\title{
Typical Retrograde Type A Dissection After Previous Type B Dissection
}

\author{
Zeyi Cheng, $\mathbf{M D}^{1}$ Jun Shi, MD, ${ }^{1}$ Caixia Pei, ${ }^{2}$ Yingqiang Guo, $\mathrm{MD}^{1}$ \\ ${ }^{1}$ Department of Cardiovascular Surgery, West China Hospital, Sichuan University, Sichuan, China; \\ ${ }^{2}$ The First Clinical Medical College of Lanzhou University, Lanzhou, Gansu, China
}

\section{ABSTRACT}

Background: Stanford type B aortic dissection (TBAD) retrograde tears to Stanford type A AD (RTAAD) have been reported rarely, fatal. Early diagnosis and timely surgery are essential. We present a typical case of RTAAD after the tip of the stent directly damaged the ascending aorta wall.

Case: A 71-year-old woman was admitted to our department for chest pain and back pain for 10 hours. She had undergone coated stent graft implantation surgery a month previously for TBAD. On first impression, we suspected the $\mathrm{AD}$ may have progressed or torn retrogradely. RTAAD was confirmed by computed tomography angiography, and we successfully performed open surgery.

Conclusion: RTAAD should be suspected in patients with chest and back pain after endovascular stent repair. Prompt recognition is essential, and early surgical treatment is strongly recommended.

\section{INTRODUCTION}

The Stanford classification of aortic dissection (AD) provides physicians with a convenient clinical method to cope with $\mathrm{AD}$. Type A AD (TAAD) needs surgical treatment, and TBAD requires primarily medical management. The use of thoracic endovascular aortic repair (TEVAR) to treat TBAD has matured and has a high success rate, but endovascular repair can also cause some harmful complications, such as paraplegia, stroke, stent migration, neurologic deficits, and retrograde tears to Stanford type A AD (RTAAD) [Khoynezhad 2013; Canaud 2014].

TBAD retrograde tears to TAAD have been reported only rarely, at an incidence of about $7 \%$ to $20 \%$. Based on TEVAR for type B dissection in 650 patients, the incidence

Received March 16, 2021; received in revised form April 19, 2021; accepted May 2, 2021.

\section{Z. Cheng and F. Shi contributed equally.}

Correspondence: Yingqiang Guo, Department of Cardiovascular Surgery, West China Hospital, Sichuan University, No. 37, Guoxue Alley, Wubou District, Chengdu, Sichuan 610041, China; 86-1898060 1876 (e-mail: drguoyq@ botmail.com). of postprocedure RTAAD was 3.38\% [Khoynezhad 2013; Canaud 2014; Kim 2014], but the mortality rate was 33\% to 57\% [Canaud 2014; Kim 2014; Canaud 2014]. Although most acute TAADs feature a tear in the aortic root or ascending aorta with antegrade propagation of the false lumen, RTAAD originates from a primary tear in the aorta beyond the left subclavian, where the false lumen propagates in a direction opposite to the direction of blood flow to the proximal arch and ascending aorta [Nauta 2016].

The key risk factors for RTAAD after TEVAR are as follows: (1) Direct injury occurs during the intervention procedure; (2) the stent graft is too big; or (3) pathological changes occur in the aortic wall itself. The specific mechanism may not be clear, but early diagnosis and timely open surgery are life-saving. We report a typical case of RTAAD after TEVAR that was successfully treated by Bentall operation and total aortic arch replacement, as well as descending aortic elephant trunk implantation.

\section{CASE PRESENTATION}

\section{Diagnosis}

A 71-year-old woman was admitted to our department for chest pain and back pain for 10 hours. About a month earlier, she underwent coated stent graft implantation surgery in our hospital for TAAD (Figure 1A). It seems that the angle of the tip of the stent was not parallel to the aortic wall, so we immediately performed computerized tomography angiography (CTA), which showed ascending aortic dissection involving the right common carotid artery and the brachiocephalic trunk (Figure 1B; Video 1). Echocardiography indicated ascending aorta dilation, mild aortic valve regurgitation, and aortic intimal tear at the distal end of sinus canal junction (Figures 1C and 1D). The patient provided written informed consent for publication of this case report and the associated images.

\section{Surgery}

The patient received surgical treatment with the support of extracorporeal circulation. Median sternotomy was performed with femoral artery and femoral vein cannulation, cardiopulmonary bypass, antegrade cardiac perfusion, and myocardial protection with cold blood cardioplegia. Transesophageal echocardiography indicated moderate aortic valve regurgitation. Antegrade and retrograde cerebral blood perfusion 
strategies were applied. Multiple ruptures of the ascending aorta around the opening of the stent could be seen intraoperatively (Figures $1 \mathrm{E}$ and $1 \mathrm{~F}$, arrow). The stent in the aortic arch was completely removed, and part of the stent in the descending aorta was cut off (Figure 1G). We performed the Bentall procedure and total aortic arch replacement, as well as descending aortic elephant trunk implantation (Figure 1H). We took care that the distal end of the aortic arch avoided the junction area between the arch and the descending aorta as much as possible: because this location is originally the junction of the 2 tissue forms, it may be difficult to suture, or postoperative aortic wall tearing may form a new dissection.

\section{Recovery}

After the operation, the patient was transferred to the intensive care unit. Adrenaline, norepinephrine, and esmolol were administered, and diuretics were used to control blood pressure $(<120 \mathrm{mmHg})$ and heart rate (60 to $90 \mathrm{bpm})$. Once heart function improved, tracheal intubation was removed, 7 hours after surgery. The patient was discharged on 10th day after surgery. Postoperative CTA showed that the artificial graft was working well (Figure 1I; Video 2), and echocardiography confirmed no aortic regurgitation.

\section{DISCUSSION}

Our patient initially presented with chest pain and back pain. The diagnosis of RTAAD was made by transesophageal echocardiography and CTA, which showed a visible entry tear and a classic dissection flap, with organized thrombus within the false lumen. During the operation, we saw several small breaches in the aortic wall all around the tip of the stent, so we believe that the RTAAD of this patient was caused by the direct damage of the stent tip to the aortic wall. By highlighting this case, we hope to remind cardiovascular interventionists that they should repeatedly confirm in TEVAR surgery whether the position of the stent tip is correct.

Acute TAAD is typically presented by antegrade $\mathrm{AD}$ from a primary intimal tear in the proximal ascending aorta extending to the arch and the downstream distal aorta. In most cases, emergency aortic replacement surgery is considered life-saving for this situation, because the natural course of untreated acute TAAD is poor. Acute TAAD may also occur in a retrograde fashion by a primary intimal tear located in the descending aorta, resulting in retrograde extension of the $\mathrm{AD}$ into the ascending aorta. The history of acute $\mathrm{AD}$ has taught us that untreated $\mathrm{AD}$ in the ascending aorta, whether antegrade or retrograde, may finally involve the coronary anatomy and the aortic valve and even cause aortic rupture.

Although Stanford type B retrograde tears to Stanford type $\mathrm{A} \mathrm{AD}$ have been reported previously, the specific mechanism is still unclear. It may be related to the aortic vessel itself or the surgical procedure, as well as device factors. Gorlitzer et al [2012] found that most patients who suffered an RTAAD after TEVAR had a primary intimal tear located at the distal convexity of the aortic arch. Al-Kalei et al [2018] also showed that patients with primary intimal tears located in the greater distal aortic arch curvature had 4 times the incidence of RTAAD after TEVAR.

Some studies have emphasized that proximal bare-spring stent grafts may increase the risk of RTAAD [Al-Kalei 2018; Kpodonu 2008; Williams 2012; Chen 2017], especially in patients with primary intimal tears located in the distal convexity. Stent grafts with proximal bare metal spring tips that open without constraint could potentially bring about a new intimal tear, so many researchers suggest avoiding using this type of stent in $\mathrm{AD}$ to reduce this fatal complication. The angle of the aortic arch and the straight structure of the stent may increase the shear stress pressing against the aortic wall, causing new intimal tears [Dong 2010].

The timing of RTAAD after TEVAR is uncertain, so it is essential to closely follow up after TEVAR to detect RTAAD. Early postoperative occurrences are related to high mortality and the need for emergent aortic replacement. As for the method of surgery, it should be determined comprehensively based on both preoperative examination and intraoperative exploration. There is no fixed surgical strategy [Williams 2012; An 2018; Neuhauser 2005]

Identifying retrograde $\mathrm{AD}$ as early as possible is essential. First, find out if the patient has a history of TEVAR. Second, ask in detail whether the patient has the following symptoms: acute chest pain, dyspnea, stroke, hypotension, syncope, etc. Third, careful physical examination is necessary. Echocardiography and CTA should be performed when necessary; intravascular ultrasound has almost $100 \%$ diagnostic accuracy. If RTAAD is highly suspected, it is very important to complete imaging as soon as possible to avoid a missed diagnosis.

The risk factors for RTAAD are summarized as follows.

- Patient factors: uncontrolled high blood pressure and heart rate, early vigorous exercise after TEVAR, Marfan syndrome, atherosclerosis, aortic malformation, or aortic aneurysm. RTAAD occurs more frequently in patients with larger false lumens. Yammine et al [2019] confirmed that the proximal landing zone and an ascending diameter $>4 \mathrm{~cm}$ are risk factors for the development of RTAAD; they also found that the presence of partial or complete false lumen thrombosis and renal ischemia are key risk factors for the development of RTAAD.

- Stent graft factors: Some studies have suggested that proximal bare stent configuration is associated with an increased risk of RTAAD [Neuhauser 2008; Dong 2009]; the more the endograft is bent, the higher the stress might be. Dong et al [2009] reported that $91.7 \%$ of patients retear in the great curvature, which is consistent with the route of the spring-back movement of the endograft. During the cardiac cycle, the dynamic movement of the ascending aorta may lead to a strong interaction with the rigid stent edge, which may also cause intimal injury with subsequent dissection. Kpodonu et al [2008] pointed out that extensive radial force due to an oversized endograft ( $>20 \%$ of the diameter of the aorta) has also been proposed as a potential cause of post-TEVAR RTAAD, but this conclusion was denied by Chen et al [2017] because there is no uniform data format for the measurement of aortic diameter. 


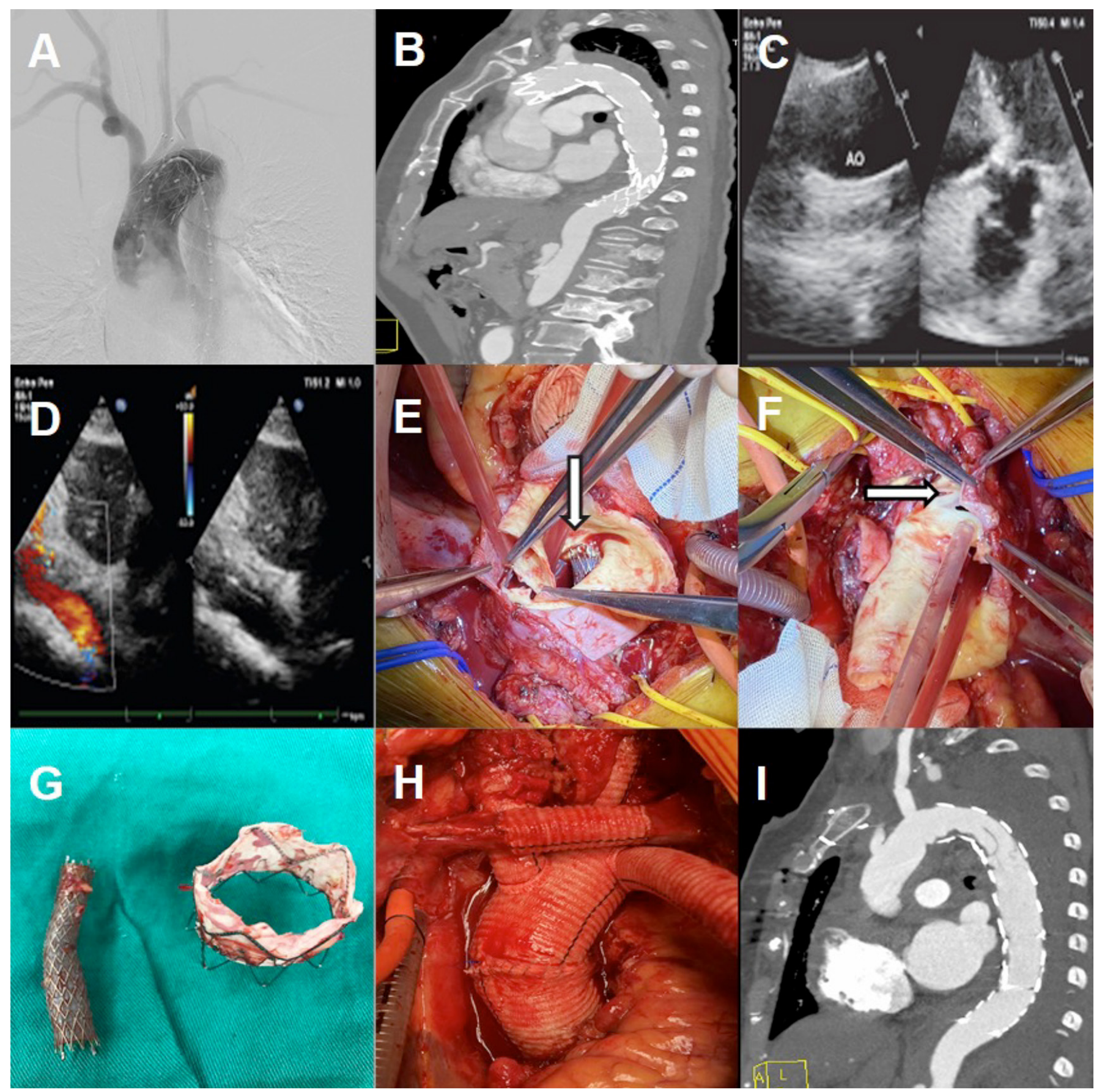

Figure 1. A, Angiography shows that the angle of the tip of the stent is not parallel to the aortic artery. B, CTA demonstrates ascending aortic dissection. $C$, Echocardiography indicates ascending aorta dilation. D, Mild aortic valve regurgitation and aortic intimal rupture at the distal end of sinus canal junction. $\mathrm{E}$, The tip of the stent can be seen perioperatively. F, In the ascending aorta, several ruptures can be seen, just in line with the tip of the stent. G, Removed stents. $\mathrm{H}$, Bentall operation and total aortic arch replacement. I, Postoperative CTA reveals that the artificial blood vessel works well. 
- Surgery methods, including guidewire, catheter, balloon dilation, delivery system, etc.

- Physician factors: centers less experienced in endovascular surgery may have a higher incidence of RTAAD [Chen 2017].

How can we reduce the incidence of RTAAD after TEVAR? Carefully select patients and devices as well as procedural components; ensure appropriate perioperative heart rate control and antihypertensive therapy; standardize the stent transplantation operation; minimize catheter and wire manipulation of the descending aorta and aortic arch in the proximal thoracic aorta; avoid balloon dilation as much as possible; and use dissection-specific devices.

\section{CONCLUSION}

We can learn lessons from this typical case that when performing TEVAR surgery, we should carefully confirm the stent's position and the angle between the tip of the stent and the wall of the aorta, especially to avoid the situation where the tip of the stent damages the aortic wall. In turn, RTAAD should be suspected in patients with chest and back pain after TEVAR surgery. Once RTAAD is diagnosed, early surgical treatment is strongly recommended. In this case, the cause of the RTAAD was likely direct damage by the stent tip to the aortic wall. Every effort should be made to place the proximal endograft where the aorta is normal to reduce possible complications.

\section{ACKNOWLEDGMENTS}

This study was supported by the National Natural Science Foundation of China (no. 81970345).

\section{[H I]REFERENCES}

Al-Kalei AMA, Wu Z, Zhang H. Influence of primary intimal tear location in type $\mathrm{B}$ aortic dissection as a factor portending retrograde type A aortic dissection after endovascular repair. J Vasc Interv Radiol 2018;29:833-840.e2.
An Z, Song Z, Tang H, Han L, Xu Z. Retrograde type A dissection after thoracic endovascular aortic repair: Surgical strategy and literature review. Heart Lung Circ 2018;27:629-634.

Canaud L, Ozdemir BA, Patterson BO, et al. Retrograde aortic dissection after thoracic endovascular aortic repair. Ann Surg 2014;260:389-395.

Chen Y, Zhang S, Liu L, et al. Retrograde type A aortic dissection after thoracic endovascular aortic repair: A systematic review and meta-analysis. J Am Heart Assoc 2017;6:e004649.

Dong ZH, Fu WG, Wang YQ, et al. Retrograde type A aortic dissection after endovascular stent graft placement for treatment of type B dissection. Circulation 2009;119:735-741.

Dong Z, Fu W, Wang Y, et al. Stent graft-induced new entry after endovascular repair for Stanford type B aortic dissection. J Vasc Surg 2010;52:1450-1457.

Gorlitzer M, Weiss G, Moidl R, et al. Repair of stent graft-induced retrograde type A aortic dissection using the E-vita open prosthesis. Eur J Cardiothorac Surg 2012;42:566-570.

Khoynezhad A, White RA. Pathogenesis and management of retrograde type A aortic dissection after thoracic endovascular aortic repair. Ann Vasc Surg 2013;27:1201-1206.

Kim JB, Choo SJ, Kim WK, et al. Outcomes of acute retrograde type A aortic dissection with an entry tear in descending aorta. Circulation 2014;130: S39-S44.

Kpodonu J, Preventza O, Ramaiah VG, et al. Retrograde type A dissection after endovascular stenting of the descending thoracic aorta. Is the risk real? Eur J Cardiothorac Surg 2008;33:1014-1018.

Nauta FJ, Tolenaar JL, Patel HJ, et al. Impact of retrograde arch extension in acute type $\mathrm{B}$ aortic dissection on management and outcomes. Ann Thorac Surg. 2016;102:2036-2043.

Neuhauser B, Czermak BV, Fish J, et al. Type A dissection following endovascular thoracic aortic stent-graft repair. J Endovasc Ther 2005;12:74-81.

Neuhauser B, Greiner A, Jaschke W, et al. Serious complications following endovascular thoracic aortic stent-graft repair for type B dissection. Eur J Cardiothorac Surg 2008;33:58-63.

Williams JB, Andersen ND, Bhattacharya SD, et al. Retrograde ascending aortic dissection as an early complication of thoracic endovascular aortic repair. J Vasc Surg 2012;55:1255-1262.

Yammine H, Briggs CS, Stanley GA, et al. Retrograde type A dissection after thoracic endovascular aortic repair for type B aortic dissection. J Vasc Surg 2019;69:24-33. 